PHARMACOLOGICAL CHAPERONES

\section{A new lens on cataract Science 350, 674-677 (2015)}

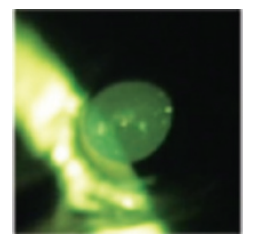

A hallmark of eye lens cataracts is the presence of aggregated crystallin proteins, a class of molecular chaperone that helps maintain the solubility of other lens proteins. To identify small-molecule chaperones, Makley et al. screened a 2,450-member compound library for those that that could inhibit the formation of the disease-linked crystallin amyloid. Potential candidates showed a decrease in the apparent melting transition temperature of the crystallin domain-containing protein Hsp27 on differential scanning fluorimetry. Over onethird of the confirmed active compounds were sterols, which led the authors ultimately to focus on 5-cholesten-3b,25-diol (compound 29) as their lead compound. NMR and in silico docking experiments suggested that compound 29 contacts both subunits of one of the cataract-related crystallin proteins, cryAB, to potentially stabilize its native state. Compound 29 could suppress amyloid formation, reverse the insolubility of the cataract-linked R120G cryAB mutant and partially disaggregate pre-formed amyloid. Compound 29 also improved lens transparency in two mouse models of hereditary cataract and in ex vivo-treated lens material from human patients with cataracts, increasing the amount of total soluble protein in both mouse tissue and human samples. These results suggest a new approach to cataract treatment using pharmacological chaperones. $M B$

MICROSCOPY TECHNIQUES

A localization module Dev. Cell 35, 513-525 (2015)

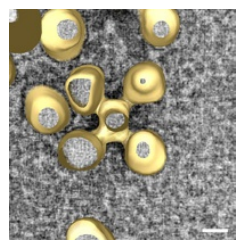

Protein tagging and protein detection with antibodies are mainstays in the toolbox for visualizing the localization of a protein of interest (POI), but the requirement to either tag each POI or use specific antibody reagents is a bottleneck for the general application of the techniques. Based on the recent demonstration of a peroxidasebased detection strategy (APEX tagging), Ariotti et al. developed a modular system for enzyme-based protein tagging. In APEX tagging, a peroxidase is appended to a POI to allow detection by light microscopy and EM of an electron-dense polymer formed by the enzyme. In the current work, the authors generated a construct containing APEX attached to a green fluorescent protein (GFP)-binding peptide (APEXGBP) and then used this to label cells that express GFP-tagged POIs, verifying their known localization to various subcellular

ANTIBACTERIALS

\title{
Stressing out dormancy
}

The difficulty of targeting dormant Mycoplasma tuberculosis (Mtb) strains remains an obstacle in tuberculosis (TB) treatment. One potential strategy is to impair the bacteria's ability to cope with elevated levels of reactive oxygen species (ROS). The sulfate assimilation pathway utilizes inorganic sulfate to produce reduced sulfur metabolites, such as cysteine and the antioxidant mycothiol, to deal with increased oxidative stress. The first step of this pathway is mediated by APS reductase (APSR), which presents a potential target for therapeutics given that Mtb CysH mutant strains, which lack APSR activity, show reduced virulence in a mouse model of TB. Palde et al. have designed a high-throughput screen using a luminescent reporter to identify inhibitors of APSR activity. They focused on analogs of the plant alkaloid ellipticine, which had strong bactericidal activity against non-replicating wild-type and multi-drug-resistant clinical isolates due to increased oxidative stress. Unlike ellipticine, these derivatives did not form DNA adducts and were nontoxic to mammalian cells at bactericidal concentrations. Palde et al. confirmed the on-target activity of these compounds by demonstrating a lack of responsiveness in $\mathrm{Cys} H$ mutant strains and showing that compound treatment decreased the production of downstream metabolites such as cysteine and mycothiol. Overall, given that APSR lacks a human homolog, these findings offer a new therapeutic avenue for the treatment of persistent and drug-resistant tuberculosis.

compartments, such as endosomes and nuclei. The method also tracks protein redistribution over time, as demonstrated for a protein that progressively moves from lipid droplets to multivesicular bodies, and in large-scale screening applications, as shown using a library of phosphoinositide-binding peptides. A linescan analysis could quantitatively assess protein enrichment at various subcellular sites. Electron tomography could also be integrated with the approach, enabling high-resolution three-dimensional analyses. These advances, as well as the application of APEX-GBP for in vivo localization of zebrafish proteins, suggest that the system could find widespread utility in rapid protein localization efforts.

CANCER THERAPY

\section{Vitamin C attack}

Science doi:10.1126/science.aaa5004

Dehydroascorbate (DHA) is the oxidized form of vitamin $\mathrm{C}$ that is taken up into cells through the glucose transporter GLUT1. Once inside the cell, DHA undergoes glutathione-mediated reduction to vitamin C. BRAF and KRAS mutant colorectal cancer cells exhibit metabolic features, such as elevated GLUT1 levels, that might make them uniquely sensitive to increased cellular uptake of vitamin C that would deplete available glutathione. Confirming this hypothesis, Yun et al. observed increased cell death of BRAF and KRAS mutant cells and mouse xenografts upon vitamin $C$ treatment due to increased reactive oxygen species (ROS) and reduced glutathione levels. Metabolomics analysis of vitamin C-treated mutant cells revealed enhanced pentose phosphate pathway flux to restore the NADPH and glutathione levels depleted by oxidation of DHA. In addition, enhanced ROS levels decreased glyceraldehyde 3-phosphate dehydrogenase (GAPDH) activity owing to increased S-glutathionylation of the active site cysteine and reduced availability of the GAPDH substrate, NAD ${ }^{+}$. Decreased GAPDH activity results in a buildup of glycolytic intermediates upstream of GAPDH and depletion of ATP, resulting in increased cellular stress. Consistent with the disruption in redox homeostasis, the addition of an antioxidant, $\mathrm{N}$-acetylcysteine, rescued vitamin $\mathrm{C}$-mediated cell death. Overall, these findings reveal an interesting mechanistic rationale explaining the sensitivity of KRAS and BRAF mutant cells to vitamin C. 